\title{
Exploring adherence to daytime compression in women with breast cancer related lymphedema: a multi-methods study
}

\author{
Mona Al Onazi', Naomi Dolgoy', Joanna Parkinson'1, Margaret L. McNeely² \\ 'Department of Physical Therapy, University of Alberta, Edmonton T6G 2G4, Canada. \\ ${ }^{2}$ Department of Physical Therapy, University of Alberta \& Cross Cancer Institute, Edmonton T6G 1Z2, Canada.
}

Correspondence to: Prof. Margaret L. McNeely, Department of Physical Therapy, University of Alberta \& Cross Cancer Institute, 2-50 Corbett Hall, Edmonton T6G 2G4, Canada. E-mail: mmcneely@ualberta.ca

How to cite this article: Al Onazi M, Dolgoy N, Parkinson J, McNeely ML. Exploring adherence to daytime compression in women with breast cancer related lymphedema: a multi-methods study. Plast Aesthet Res 2020;7:23.

http://dx.doi.org/10.20517/2347-9264.2019.74

Received: 16 Dec 2019 First Decision: 27 Mar 2020 Revised: 3 Apr 2020 Accepted: 15 Apr 2020 Published: 11 May 2020

Science Editor: Xiao Long Copy Editor: Jing-Wen Zhang Production Editor: Jing Yu

\begin{abstract}
Aim: The objective of this follow-up study was to explore the barriers and facilitators to use of daytime compression among women with breast cancer related lymphedema who previously took part in a trial examining the efficacy of night compression.
\end{abstract}

Methods: We used a multi-methods approach involving a survey and subsequent focus group sessions. The survey questions were developed based on clinical experience and findings from the literature. Questions were framed to align with the Theoretical Domains Framework. For the focus group data, we applied an interpretive description qualitative methodology to understand participants' experiences and views on use of daytime compression. Qualitative findings were mapped to the Theoretical Domains Framework.

Results: Questionnaires were completed by 48 of 52 participants. Only 15 participants (31\%) reported adhering to wearing the garment for greater than $12 \mathrm{~h}$ each day. Better adherence was positively associated with perceived control of lymphedema ( $r=0.304 ; 95 \% \mathrm{Cl}: 0.051-0.564 ; P=0.021)$. Survey findings suggest that participants have good knowledge about the rationale for, and the benefits of, wearing the compression sleeve. Twentythree survey respondents took part in one of the five subsequent focus group sessions. Five key themes were identified representing the primary barriers to regular use of daytime compression: discomfort, negative emotions, interference with function, social situations and visibility, and use of alternative management strategies.

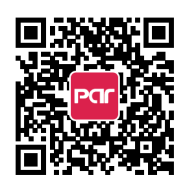


Conclusion: The findings suggest less than optimal adherence to daytime compression sleeve use. Further research is needed to explore the relative benefit of daytime compression, optimal wear times, and implementable strategies to improve adherence.

Keywords: Lymphedema, breast cancer, compression therapy, adherence

\section{INTRODUCTION}

Lymphedema, a significant swelling of the arm that occurs on the side of the breast cancer, is one of the more frequent complications following surgical interventions for breast cancer ${ }^{[1,2]}$. It is a lifelong condition that tends to worsen over time ${ }^{[1,3,4]}$. Recent data suggest that approximately $21 \%$ of women who undergo treatment for breast cancer are diagnosed with lymphedema ${ }^{[1]}$. Of these cases, approximately half will develop chronic progressive lymphedema, a more severe presentation of ongoing and complex swelling ${ }^{[2,5]}$.

At present, there are no known curative treatments, either surgical or pharmacological, for lymphedema ${ }^{[6,7]}$. Conservative treatments are prescribed to reduce and maintain limb size, restore function, reduce pain, and improve the appearance of the limb ${ }^{[6,8,9]}$. Decongestive lymphatic therapy, often prescribed to reduce the lymphedema volume, is an intensive treatment program usually delivered in a clinical or hospital setting ${ }^{[9]}$. This program is followed by a maintenance phase which aims to promote life-long control of lymphedema through use of self-management strategies ${ }^{[9,10]}$. Currently, daytime compression sleeves represent the standard of care and are the primary strategy in lymphedema maintenance ${ }^{[6]}$.

We conducted a trial examining the efficacy of the addition of nighttime compression to standard care, involving daytime use of a compression sleeve, on arm lymphedema volume (LYNC trial) ${ }^{[11]}$. The LYNC trial was a parallel three-arm, multicenter randomized fast-track trial involving three sites in Canada: (1) the Cross Cancer Institute in Edmonton; (2) Tom Baker Cancer Centre in Calgary; and (3) University of British Columbia in Vancouver ${ }^{[11]}$. One hundred twenty women enrolled in the trial and were randomly assigned to one of three groups: (1) standard care (daytime use of a compression sleeve alone); (2) standard care plus nighttime compression through self-administered or assisted compression bandaging; and (3) standard care plus nighttime compression through use of a nighttime compression system garment ${ }^{[1]}$. At the end of the randomized controlled trial portion of the study, participants in the two comparison groups were provided with a nighttime compression system garment and followed the night regimen for an additional three months ${ }^{[11]}$.

During the LYNC study, all participants were asked to follow standard care recommendations that involved wearing their daytime compression sleeve daily for a minimum of $12 \mathrm{~h}$. We monitored adherence to use of daytime compression among participants and found that, while overall adherence (based on hours worn per week) was $81 \%$, only $58 \%$ of participants adhered to the recommendations of seven days per week and $12 \mathrm{~h}$ per day. In contrast, mean adherence to nighttime compression during the trial was $100 \%$ both during the intervention and follow-up periods of the randomized controlled trial. As adherence to use of daytime compression is seen as a critical factor in long-term lymphedema control ${ }^{[12]}$, we aimed to explore the reasons participants reported less than optimal adherence to use of their daytime compression. Thus, the objective of this follow-up study was to explore the barriers and facilitators to use of a daytime compression sleeve.

\section{METHODS}

\section{Study design}

The study used a multi-methods approach involving quantitative and qualitative survey data. In this design, we conducted both a survey and five focus group sessions to collect information from participants on their perspectives and experiences with use of daytime compression. 


\section{Conceptual framework}

The survey questions were developed based on clinical experience of the researchers and findings from the literature around compression therapy adherence. Questions were then framed to align with the Theoretical Domains Framework (TDF). The TDF is a behavior change model used in the field of implementation science to inform healthcare practitioner behavior ${ }^{[13,14]}$. The TDF comprises 14 theoretical domains that are considered to influence behavior and behavioral change ${ }^{[15]}$. Used in conjunction, the TDF and the Behavior Change Wheel (BCW) link the identified determinants of behavior to appropriate behavior change techniques. At the center of a proposed BCW is a behavior system involving three essential conditions: capability, opportunity, and motivation $(\mathrm{COM}-\mathrm{B})^{[15]}$. These conditions interact to produce the behavior ${ }^{[16]}$. Capability is defined as the individual's psychological and physical capacity, which includes having the necessary knowledge and skills to perform the behavior ${ }^{[16]}$. Motivation is defined as the intellectual processes that energize and direct behavior, which includes goals, habits, emotional responses, and decision-making ${ }^{[16]}$. Opportunity is defined as the external influences that impact the individual, making possible or encouraging the occurrence of the behavior ${ }^{[16]}$.

Together, the TDF and BCW allow mapping of behavior change techniques to tailored implementation strategies ${ }^{[13]}$. Although originally developed for use with healthcare practitioners, the TDF is now commonly used to behavior change across patient populations ${ }^{[17]}$.

The survey consisted of 45 questions in six categories: demographic, health and lymphedema status, garment use, garment knowledge, number and cost of garments, and social and environmental factors. After development and initial testing for clarity and effectiveness with the research team and clinic staff, the survey was pilot tested with two women with breast cancer related lymphedema (BCRL) who were not part of the LYNC study [Supplement Table 1].

\section{Qualitative methodology}

For the purpose of this study, we applied an interpretive description (ID) qualitative methodology to understand participants' experiences and perspectives on the use of daytime compression as a selfmanagement strategy for lymphedema ${ }^{[18]}$. ID can be applied to qualitative inquiry, as a means to better understand individual health and illness, and to generate useful knowledge that can be used in clinical practice $^{[18,19]}$. Further, ID used in focus groups offers a methodological approach to the discovery and creation of shared perspectives, rather than simply relying on individual reports ${ }^{[18]}$.

\section{Participant sampling}

A convenience sample of 93 women who participated in the LYNC study within Alberta (Edmonton and Calgary) was screened for eligibility through the electronic medical records system.

Eligibility for the survey included: (1) prior participation and completion of the LYNC study; (2) current residence in Alberta, Canada; and (3) stable breast cancer medical status.

Eligible women were invited via email or phone to participate in the survey. Participants agreeing to the survey had the option to complete the survey online through REDCap ${ }^{[20,21]}$ (a secure web application for building and managing online surveys) or to receive a paper copy through regular mail.

\section{Data collection}

Findings from the surveys were used to develop the probing questions for the focus group sessions. The last question of the survey asked participants if they were willing to take part in a focus group session. Participants indicating interest were then contacted and booked for a session, either at the University of Alberta in Edmonton or at the Holy Cross Centre in Calgary. The focus group sessions took approximately $90 \mathrm{~min}$ each, and were simultaneously audio recorded and manually transcribed. The principal investigator 
(MM) facilitated the focus group discussions at both locations, with a second researcher in attendance at each session in Edmonton (MAO) and Calgary (ND).

\section{Data analysis}

The survey results were analyzed quantitatively using the means and standard deviations for continuous variables, and frequencies and percentages for nominal variables. We also examined the association between adherence to daytime compression and perceived control of lymphedema, overall and by lymphedema severity, using a Chi square correlation coefficient. All focus group tapes were audio recorded and transcribed verbatim by the research team. ID was used to guide the process of capturing patterns and themes about perceptions and perspectives related to the participants' adherence to daytime compression. Data analysis was based on a thematic analytic approach and followed the processes as described by Braun and Clarke ${ }^{[22]}$. The data were examined line by line by the researchers independently to identify patterns and key themes with sample quotations from the data. Codes for similar meanings and highlighted terms were developed, examined, and refined as necessary to identify the most expressive codes to represent the participants' voice. The researchers then discussed and reviewed the independently coded data together, until a consensus on themes was reached. Conceptual themes were inductively originated from the analysis, by first generating initial codes, followed by subthemes, and finally by generating an overarching theme ${ }^{[22]}$. An additional step was taken to map the qualitative data back to the TDF. Any responses or comments that did not answer the specific question were not included in the analysis.

\section{Ethical considerations}

Ethical approval was obtained from the Health Research Ethics Board of Alberta: Cancer Committee for this additional follow-up component of the LYNC study. All participants provided amended informed consent. Each participant was coded by a study number to protect her identity.

\section{RESULTS}

Questionnaires were completed by 48 of 52 participants who responded to our invitation to take part in the survey, for an overall completion rate of $92 \%$ [Figure 1]. Twenty-three of the survey respondents (48\%) took part in one of the five subsequent focus group sessions. Four focus group sessions were held in Edmonton ( $n$ $=18)$ and one was held in Calgary $(n=5)$.

\section{Quantitative survey results}

The mean age of participants was 65 years (range 39-82). The majority of participants (80\%) described their general health as "good" or "very good". More than half of the participants reported a duration of lymphedema of greater than six years (58\%), and that the extent of their swelling was "moderate" (69\%). Most commonly, participants reported the status of their lymphedema as "well controlled" (38\%) or "fluctuating" (44\%), with $34 \%$ stating they were bothered by their lymphedema "moderately" or higher. Of the 12 participants (25\%) reporting "slight/mild" swelling, only one (5\%) stated she was bothered by her lymphedema more than "a little bit" [Table 1].

Twenty-eight participants (58\%) reported using their sleeve daily, and 17 (35\%) reported using the garment for $>12 \mathrm{~h}$ per day. When we examined adherence to both days and hours, only 15 participants (31\%) reported adhering to wearing the garment $>12 \mathrm{~h}$ every day.

Survey findings suggest that the vast majority of participants (98\%) have good knowledge about the rationale for, and the benefits of, wearing the compression sleeve [Table 2]. Forty-three participants (90\%) reported that their compression sleeve helped them to manage their lymphedema. Most participants understood the different types (85\%), compression levels (77\%), and recommended number of hours to 


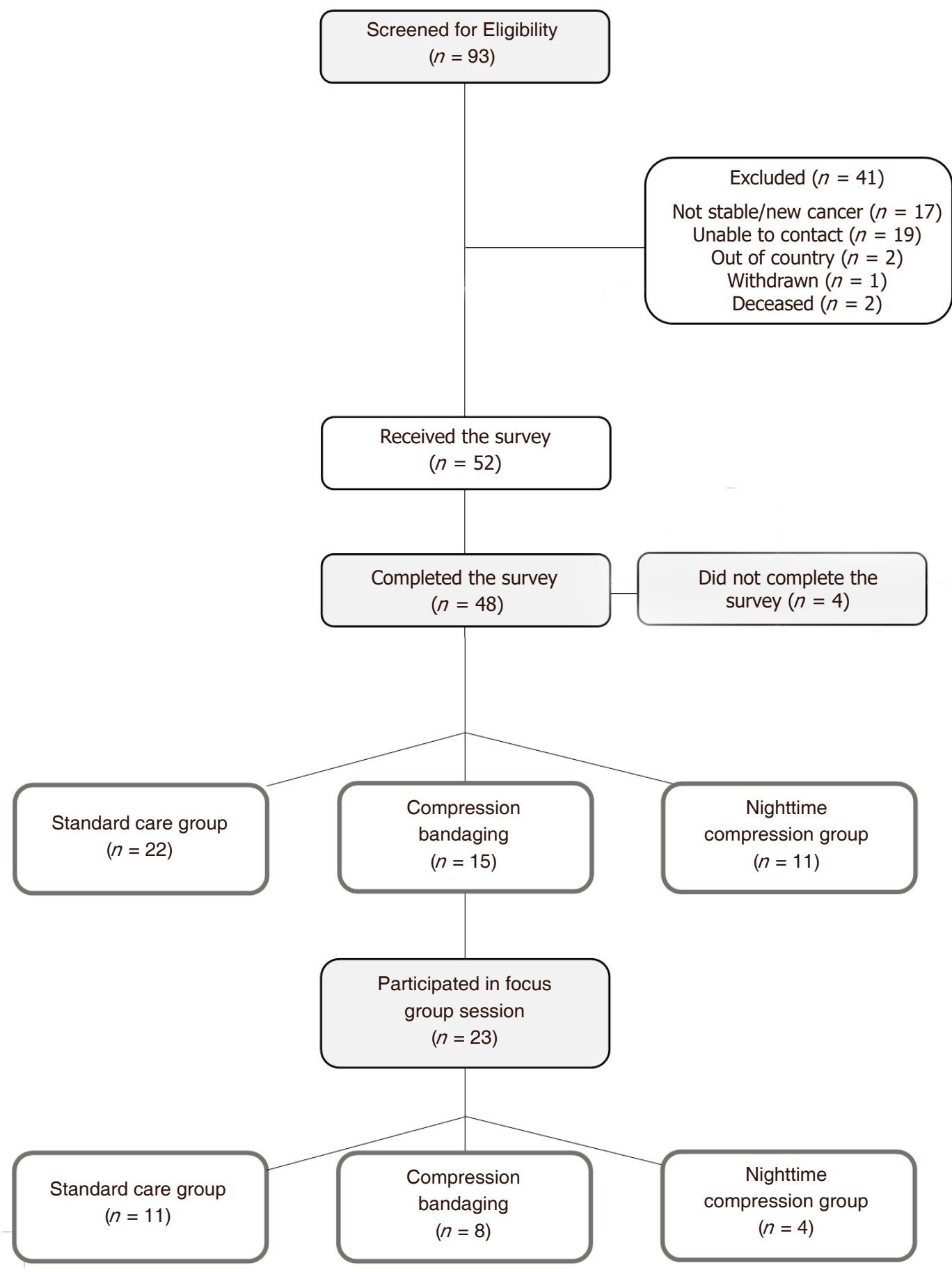

Figure 1. Flowchart of recruitment

wear the sleeve $(83 \%)$. In addition, the majority of participants reported they had the skills, confidence, and ability to don the compression sleeve [Table 2].

The main identified factors related to non-adherence were weather, social settings, and interference with function. Twenty-three participants (48\%) reported that their ability to wear the sleeve was affected by weather, with 21 (44\%) reporting wearing their sleeve less during summer season due to discomfort related to heat and humidity. Twenty-four participants (50\%) reported not wearing the compression sleeve when 
Table 1. Summary of participant characteristics $(n=48)$

\begin{tabular}{|c|c|c|}
\hline Age & Mean (range) & 65 years $(39-82)$ \\
\hline \multirow[t]{5}{*}{ Employment, $n(\%)$} & Retired & $28(58 \%)$ \\
\hline & Sick leave/disability & $1(2 \%)$ \\
\hline & Part-time & $3(6 \%)$ \\
\hline & Full-time & $14(29 \%)$ \\
\hline & Homemaker & $2(4 \%)$ \\
\hline \multirow[t]{4}{*}{ Marital status, $n(\%)$} & Married or common-law & $30(64 \%)$ \\
\hline & Single & $2(4 \%)$ \\
\hline & Divorced & $9(19 \%)$ \\
\hline & Widowed & $6(13 \%)$ \\
\hline \multirow[t]{2}{*}{ Current cancer treatments, $n(\%)$} & Hormone therapy & $13(28 \%)$ \\
\hline & None & $33(72 \%)$ \\
\hline \multirow[t]{4}{*}{ General health, $n(\%)$} & Excellent & $4(8 \%)$ \\
\hline & Very good & $19(40 \%)$ \\
\hline & Good & $19(40 \%)$ \\
\hline & Fair & $6(12 \%)$ \\
\hline \multirow[t]{3}{*}{ Lymphedema duration, $n(\%)$} & 4-5 years & $20(42 \%)$ \\
\hline & $6-10$ years & $19(40 \%)$ \\
\hline & $>10$ years & $9(19 \%)$ \\
\hline \multirow[t]{4}{*}{ Lymphedema severity, $n$ (\%) } & Slight-mild swelling & $12(25 \%)$ \\
\hline & Moderate swelling & $33(69 \%)$ \\
\hline & Severe swelling & $1(2 \%)$ \\
\hline & Very severe & $2(4 \%)$ \\
\hline \multirow[t]{4}{*}{ Lymphedema status, $n$ (\%) } & Well-controlled, stable & $18(38 \%)$ \\
\hline & Improving & $7(15 \%)$ \\
\hline & Fluctuating: sometimes better, sometimes worse & $21(44 \%)$ \\
\hline & Worsening & $2(4 \%)$ \\
\hline \multirow[t]{5}{*}{ Bothered by lymphedema, $n$ (\%) } & Not at all & $8(17 \%)$ \\
\hline & A Little bit & $19(40 \%)$ \\
\hline & Moderately & $12(25 \%)$ \\
\hline & Quite a bit & $6(13 \%)$ \\
\hline & Extremely & $3(6 \%)$ \\
\hline
\end{tabular}

attending specific social settings and 13 (27\%) reported hiding the sleeve when in public settings due to unwanted attention or questions. Fourteen participants (29\%) reported that their sleeve interfered with their work or daily routine [Table 3].

\section{Adherence and perceived control of lymphedema}

A total of 28 participants (58\%) reported wearing their sleeve every day, and, of those, $21(76 \%)$ reported their lymphedema was "well controlled or improving" $(P=0.01)$. When we compared daily adherence of > $12 \mathrm{~h}$ daily to non-adherence, there was no significant difference in self-perceived control of lymphedema $(P>0.05)$. However, when we examined daily adherence at a level of $>9$ h daily, of the 25 participants who reported adherence at $>9$ h, 17 (68\%) reported their lymphedema was "well controlled" or "improving" $(r=0.304$; 95\%CI: 0.051-0.564; $P=0.021)$. The strength of the relationship increased when we limited the analysis to only those participants $(n=33)$ with moderate lymphedema [Table 4 ].

When we explored the data based on severity of lymphedema, of the 12 participants with slight or mild swelling, 11 reported their lymphedema was well controlled or improving; however, only 6 (50\%) reported wearing their sleeve daily for $>9 \mathrm{~h}$. In contrast, of the 36 participants with moderate-to-severe lymphedema, 14 (39\%) participants reported their lymphedema was well controlled or improving, with 11 of the 14 reporting wearing their sleeve daily for $>9 \mathrm{~h}(P<0.047)$ [Table 5].

\section{Qualitative findings}

Based on the findings of survey, the following probing questions were developed for the subsequent focus group sessions, to help us to better understand the participants' perspectives on wearing a sleeve, when 
Table 2. Reported compression garment data

\begin{tabular}{|c|c|c|}
\hline \multicolumn{3}{|l|}{ Garment use } \\
\hline Garment adherence & 7 days/week & $28(58 \%)$ \\
\hline \multirow[t]{4}{*}{ Days per week } & 6 days/week & $7(15 \%)$ \\
\hline & 4-5 days/week & $6(13 \%)$ \\
\hline & 1-3 days/week & $4(8 \%)$ \\
\hline & 0 days/week & $3(6 \%)$ \\
\hline Garment adherence & 4 h or fewer & $7(15 \%)$ \\
\hline \multirow[t]{3}{*}{ Hours per day } & $5-8 h$ & $9(19 \%)$ \\
\hline & $9-12 \mathrm{~h}$ & $15(31 \%)$ \\
\hline & $\geq 12 \mathrm{~h}$ & $17(35 \%)$ \\
\hline $\begin{array}{l}\text { Garment adherence } \\
7 \text { days/week }\end{array}$ & $\geq 12 \mathrm{~h}$ & $15(31 \%)$ \\
\hline \multirow[t]{2}{*}{ Compression sleeve interfere with daily routine } & Yes & $14(29 \%)$ \\
\hline & No & $34(71 \%)$ \\
\hline \multirow[t]{2}{*}{ Wear the sleeve for house work/activities/exercise } & Yes & $42(88 \%)$ \\
\hline & No & $6(12 \%)$ \\
\hline \multicolumn{3}{|l|}{ Garment knowledge } \\
\hline \multirow[t]{4}{*}{ Topics discussed with therapist } & The compression garment types & $41(85 \%)$ \\
\hline & The compression level or degree & $37(77 \%)$ \\
\hline & Number of hours per day to wear the garment & $40(83 \%)$ \\
\hline & No discussion & $3(6 \%)$ \\
\hline \multirow[t]{2}{*}{ Compression sleeve helps to manage lymphedema } & Yes & $43(90 \%)$ \\
\hline & No & $5(10 \%)$ \\
\hline $\begin{array}{l}\text { *Understand the reason for wearing the compression sleeve } \\
\text { every day, \% (SD) }\end{array}$ & VAS (0-100) & $88 \%(16)$ \\
\hline $\begin{array}{l}\text { *Have a good knowledge about the compression sleeve } \\
\text { benefits, \% (SD) }\end{array}$ & VAS $(0-100)$ & $86 \%(15)$ \\
\hline \multirow[t]{2}{*}{ Have personal benefits from wearing the garment } & Yes & $43(90 \%)$ \\
\hline & No & $5(10.4 \%)$ \\
\hline \multirow{2}{*}{ Confident in putting on the compression sleeve properly } & Yes & $47(97.9 \%)$ \\
\hline & No & $1(2.1 \%)$ \\
\hline
\end{tabular}

*Visual analog scale (VAS): 0: not at all; 100: very much. SD: standard deviation

Table 3. Reported social and environmental factors

\begin{tabular}{|c|c|c|}
\hline \multicolumn{3}{|l|}{ Social and environmental factors } \\
\hline \multirow[t]{2}{*}{ The weather affects the compression sleeve adherence } & Yes & $23(47.9 \%)$ \\
\hline & No & $25(52.1 \%)$ \\
\hline \multirow[t]{5}{*}{ Wear it less during the following seasons } & Spring & $4(8.3 \%)$ \\
\hline & Summer & $21(43.8 \%)$ \\
\hline & Fall & $1(2.1 \%)$ \\
\hline & Winter & $1(2.1 \%)$ \\
\hline & Wear it the same throughout the year & $26(54.2 \%)$ \\
\hline \multirow[t]{2}{*}{ Wear the sleeve at home } & Yes & $39(81.3 \%)$ \\
\hline & No & $9(18.8 \%)$ \\
\hline \multirow[t]{2}{*}{ Wear the sleeve in public } & Yes & $42(87.5 \%)$ \\
\hline & No & $6(12.5 \%)$ \\
\hline \multirow[t]{2}{*}{ Hide the sleeve in public } & Yes & $13(27.1 \%)$ \\
\hline & No & $35(72.9 \%)$ \\
\hline \multirow[t]{2}{*}{ Not wearing the sleeve during some social settings } & Yes & $24(50.0 \%)$ \\
\hline & No & $24(50.0 \%)$ \\
\hline \multirow{2}{*}{$\begin{array}{l}\text { Have supportive family and friends on wearing } \\
\text { compression sleeve }\end{array}$} & Yes & $47(97.9 \%)$ \\
\hline & No & $1(2.1 \%)$ \\
\hline${ }^{\star}$ Have issues putting the garment on, \% (SD) & VAS $(0-100)$ & $16 \%(24)$ \\
\hline${ }^{*}$ Need help with sleeve donning, \% (SD) & VAS $(0-100)$ & $16 \%(28)$ \\
\hline
\end{tabular}

*Visual analog scale (VAS): 0: not at all, 100: very much . SD: standard deviation 
Table 4. Association between adherence and perceived control of lymphedema overall

\begin{tabular}{lccc}
\hline Adherence & Well-controlled or improving & Fluctuating or worsening & Total \\
\hline Daily $>9 \mathrm{~h}$ & 17 & 8 & 25 \\
$<7$ days or $<9 \mathrm{~h}$ & 8 & 15 & 23 \\
Total & 25 & 23 & 48 \\
\hline
\end{tabular}

$r=0.304 ; 95 \% \mathrm{Cl}: 0.051-0.564 ; P=0.021$

Table 5. Association between adherence and perceived control for those with moderate to severe lymphedema

\begin{tabular}{llccc}
\hline Lymphedema severity & Adherence & Well-controlled or improving & Fluctuating or worsening & Total \\
\hline Moderate & Daily $>9 \mathrm{~h}$ & 11 & 9 & 20 \\
& $<7$ days or $<9 \mathrm{~h}$ & 3 & 13 & 16 \\
& Total & 14 & 22 & 36 \\
\hline
\end{tabular}

$r=0.396 ; 95 \% \mathrm{Cl}: 0.043-0.745 ; P=0.047$

and why they do not wear the sleeve in specific circumstances (e.g., social situations and weather), and the impact of wearing the sleeve on daily function:

1. What are the benefits for you with wearing the sleeve?

2. Does anything make it easier or harder for you to wear a sleeve for 12 hours a day?

3. How do you feel about wearing your compression sleeve?

4. What is your experience/perspective on times when you don't wear your sleeve?

4.a. Do you have strategies to manage your lymphedema in these situations?

Sample supporting quotations are provided in each section to illustrate participants' perspectives.

\section{Benefits of wearing the garment}

Across all focus group sessions, the primary identified benefit of wearing the sleeve was to reduce or control the lymphedema. Other reported benefits included symptom management (e.g., reduced pain/ discomfort, tension, and heaviness in limb), protection of the skin (e.g., reduced risk of damaging the skin), preventing/reducing episodes of cellulitis, and keeping the shape/contour of the limb.

- "I am pretty good about wearing the sleeve, because I know it manages the swelling."

- "I know I need to wear my sleeve, so I do."

Facilitators to regular use of the sleeve

Facilitators to regular use of daytime compression included using adaptive strategies (e.g., using older sleeves for gardening or dirty tasks), having more garment options (e.g., colors to match outfits and lower compression garment options for exercise), and having the money or medical insurance to help cover costs of extra garments (e.g., less washing and a clean garment for each work day). The majority of participants reported that wearing their garment was a habit and that they had accepted the need to wear it.

- "It is part of my dress every day."

- "I never worry about social situations, it is who I am; never think about it."

- "I do get asked a lot by children, sometimes adults, I explain simply. My granddaughter who is 18 months keeps me honest - she notices if I haven't got it on."

- "Nice to dress it up and have fun with it. If I have to wear it, I want it to be a fashion statement!"

Barriers to regular use of the sleeve

Five key themes were identified representing the primary barriers to regular use of the compression sleeve or reasons for non-use: discomfort, negative emotions, interference with function, social situations and visibility, and alternative management strategies. 


\section{Theme: discomfort}

Discomfort was reported as a result of a number of factors including: (1) poor fit of the garment; (2) heat and humidity/weather; and (3) poor tolerance to compression. Issues related to the fit of the garments mainly included fit at the hand (e.g., irritation of thumb and web spaces), wrist (e.g., space between glove and sleeve and overlap of compression), elbow (e.g., nerve compression and chafing in elbow crease), or at the upper most aspect of arm (e.g., band too tight, top of sleeve sliding down, and blistering due to silicon band). Participants also reported that fit tended to worsen over the course of the day and when the sleeve was older.

- "My arm is full, and circulation at elbow can feel cut off if the arm is bent for prolonged periods of time."

- "I don't think I get the benefits out of it as I would if it fit well."

- "My sleeve tends to slide down, and I am constantly adjusting it."

Heat was a common factor for removing the sleeve. Participants mostly reported weather and temperature as contributors to heat-related discomfort; however, some participants reported increased heat due to hot flashes or from activity and exercise.

- "In the summer, it is way too hot to wear a sleeve and glove."

- "It's too hot, I can't even get it on in warm weather as I am too sweaty."

- "When I have a hot flash, I want to take the sleeve off."

Increased discomfort due to progressive tension/tightness in the limb was also reported as a primary reason for earlier removal of the sleeve prior to the 12 -h recommended time.

- "At the end of my work day, it feels too restrictive and uncomfortable, so I take it off."

\section{Theme: negative emotions}

A minority of participants expressed negative feelings associated with wearing the sleeve daily. The expressed feelings included words such as "hate", "guilt", and "fear"; participants reported the following concerns regarding negative emotions towards the garments:

- "I hate it. I am tired of the reminder that it is always there, and no end in sight for getting better."

- "Life changing, ruins my life to wear a sleeve, I can't do the things I want to do with a sleeve and glove."

- "When I don't wear it, I feel guilty."

- "I fear the lymphedema will get worse."

Many participants reported burdens related to wearing the sleeve daily, including laundering their garments, the need for ongoing fitting appointments, and having to get garment prescriptions renewed.

- "I get tired of the regimen, I need a break."

- "I am tired of proving I still need a sleeve, this is a lifelong condition."

- "I still need a prescription each year for insurance, this is frustrating."

Participants requiring custom compression sleeves and gloves expressed frustration with fit, issues with returning poorly fitted garments, and wait times for garments; participants expressed the following concerns with their garments:

- "Fittings are hit and miss, especially garment length."

- "When it comes wrong, I don't bother sending it back - too much of a hassle."

- "Long wait for custom garment, then it comes but does not fit well."

\section{Theme: interference with function}

The primary finding in regards to interference with function related to hand function and concerns over hygiene, especially when using a glove or gauntlet. With respect to function, participants reported the following concerns:

- "I have difficulty manipulating objects, doing crafts, and with writing due to the glove, so I remove it." 
- "I live on a farm, and I spend a lot of time taking it on and off so it will not get wet or dirty."

- "I need to feel the food with my fingers, so I can't wear the glove in the kitchen."

- "The glove gets wet with meal preparation, sleeve is uncomfortable with the glove off, so I remove both."

- "The glove is cumbersome and interferes with cooking and hand washing."

With respect to maintaining cleanliness of the garments, participants reported the following concerns with managing hygiene:

- "I work as a surgical nurse and cannot wear my garment during surgery as I cannot scrub and stay sterile, and wear the sleeve."

- "I need to wash my hands at work, so I have to remove the glove a lot."

- "I worry about germs. Is the glove carrying germs?"

\section{Theme: social situations and visibility}

When we explored reasons for non-use of the sleeve for social events or special occasions, participants reported issues with appearance and with the garments drawing unwanted attention or questions.

- Appearance:

- "When I go out for a special evening event, and when wearing an evening outfit, I will go without the sleeve."

- "I won't wear it for weddings or formal events, as it does not look good with my outfit"

- "I won't wear it for a photo session, doesn't look good."

- "Clothes don't fit well when I have it on."

- Drawing unwanted attention:

- "Lady with the sleeve."

- "I am always conscious of it because I have people asking me if I have been burned or why I wear it."

- "It draws attention to the arm and awkward questions."

- "I like fall and winter more because I can wear long sleeve shirts and nobody asks me anything."

Theme: alternative management strategies

Many of the participants reported the use of alternative strategies that helped them to control their lymphedema and allowed them to "get away" with reducing daytime compression sleeve wear. Nighttime compression, through bandaging or a night garment, was the main strategy used as an alternative to wearing daytime compression. Other strategies included managing body weight, exercise, and nutritional intake (such as avoiding alcohol and salt).

- "My night system controls my lymphedema, so I don't need to wear a daytime sleeve anymore."

- "Even minor weight loss seems to help me control the lymphedema better."

- "Exercise helps me a lot."

- "Drinking alcohol makes the swelling worse, I know I will need to wear my garment more after a social event."

- "Salt definitely makes the swelling worse, especially if I eat out."

\section{Data synthesis: mapping of facilitators and key themes to the TDF}

The findings of the qualitative study were combined with survey data and mapped to the TDF data to allow for the identification of potential strategies to enhance adherence to daytime compression. We found that the main themes could not be categorized into a single component of the COM-B model. Clearly, relationships existed among the components of opportunity, capability, and motivation, while some barriers influenced motivation. For example, not wearing the sleeve when attending social events was related to visibility of the garment with dress clothes representing social influences (opportunity), while the unwanted 


\section{FACILITATORS TO WEARING SLEEVE}

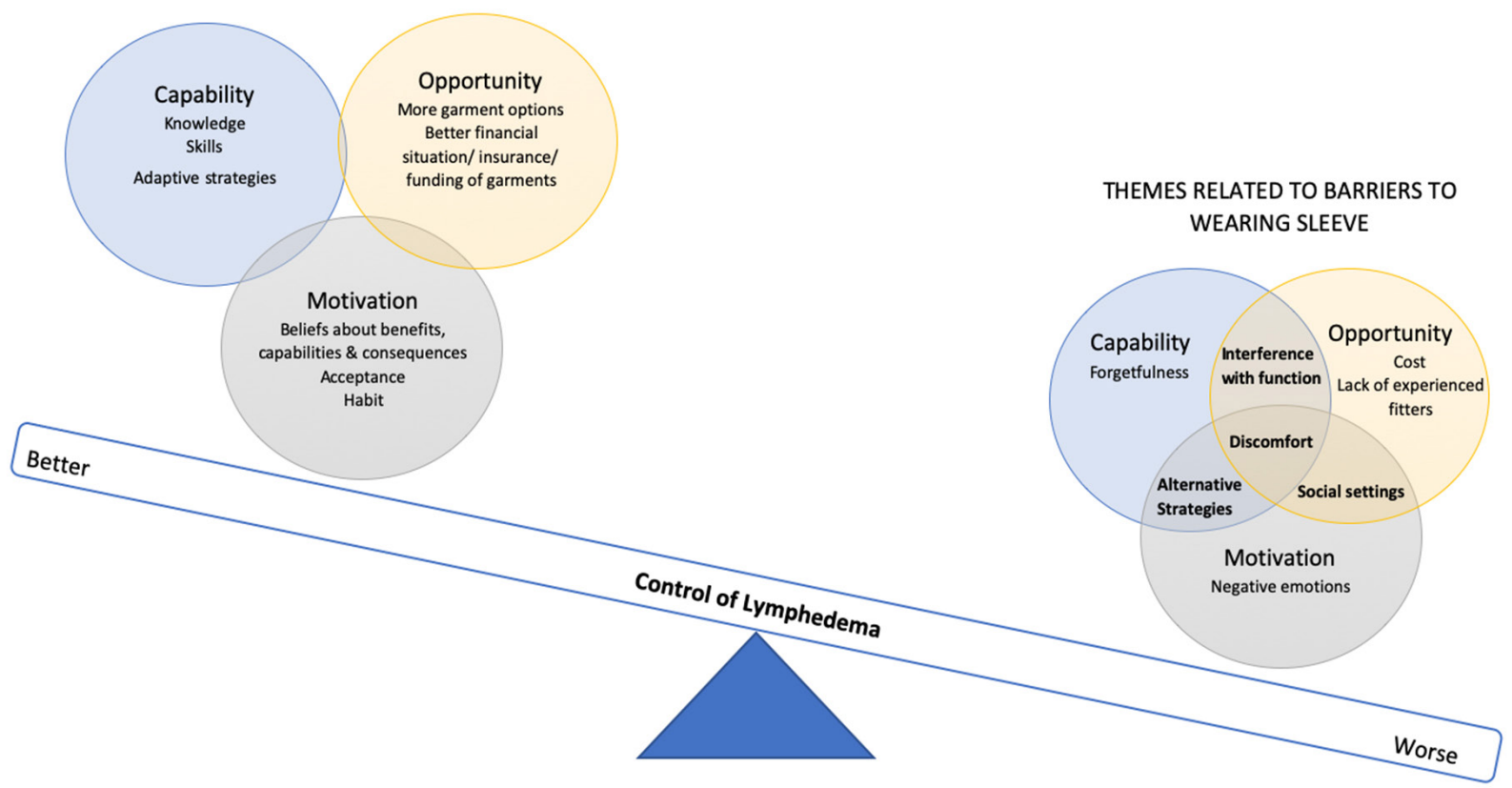

Figure 2. Survey and qualitative findings mapped to the capability, opportunity, and motivation behaviour change wheel (COM-B)

attention was related to emotion (motivation). "Discomfort" crossed components of capability (i.e., lack of adaptive strategies) and opportunity (e.g., lack of experienced fitters and environmental factors/weather) leading to issues with motivation (i.e., negative emotions). Interference with function appeared to primarily involve the lack of opportunity in relation to proper fit of the garment, and the need for garments that both facilitate functional/work activities and are hygienic. We noticed that interference with function also appeared to be influenced by a lack of adaptive strategies (capability) [Figure 2].

\section{DISCUSSION}

A primary finding of this study was that adherence to daytime compression was less than optimal. At present, compression garments are the mainstay of lymphedema self-management ${ }^{[10]}$, and are generally recommended for use during all waking hours ${ }^{[23,24]}$. Poor adherence to BCRL self-care practices has been associated with increased arm volume and progression of BCRL to more advanced stages ${ }^{[12]}$, supporting the importance of good adherence. A prior research study examining adherence to self-care strategies had similar findings, reporting that only $39 \%$ of their study participants adhered to greater than $75 \%$ of recommended compression sleeve wear time across a one-year follow-up period ${ }^{[25]}$. While overall adherence was poor among participants in our study, those participants who reported daily adherence of $>$ $9 \mathrm{~h}$ also reported better perceived-control of their lymphedema, suggesting a positive medium effect from use of daytime compression. Based on our findings, however, only $9.2 \%$ of the total variation in perceived control can be explained by adherence to use of daytime compression. Thus, $91 \%$ of the variation is related to other potential factors including the use of other maintenance strategies identified by the participants.

\section{Capability}

The results of this study suggest that strict adherence to use $>12 \mathrm{~h}$ per day, while recommended, may not be necessary to achieve control of lymphedema, especially in the case of those with mild lymphedema. Other self-management practices reported by many participants in this study were used to replace the need for strict adherence to daytime compression, by supplementing wear schedules with nighttime 
compression. This is not surprising given that all participants had previously taken part in the LYNC study and had been provided with a nighttime compression garment. In a previous cohort study, adherence to daytime compression and use of nighttime compression bandaging four nights a week was shown to result in better control of lymphedema over the longer term as compared to daytime compression alone ${ }^{[12]}$.

Other strategies reported by participants included using lower compression sleeves for activities and exercise, and older sleeves for housework and gardening. In addition, participants reported use of lifestyle strategies such as exercise, weight management, and proper nutrition to improve overall control of their lymphedema ${ }^{[26]}$. As lymphedema is a chronic condition, tailored approaches involving combinations of selfmanagement strategies may allow women more flexibility in terms of daytime compression wear time.

\section{Opportunity}

The findings of this study suggest the need for healthcare providers and patients to work with industry partners to develop options to address barriers to garment wear. For example, innovation is needed to explore options for garments that facilitate activity participation, such as more breathable garment fabrics to address discomfort related to heat; waterproof and more hygienic options for gloves; and garments that match skin tones to reduce visibility of the sleeve. There is also a clear need for increased training and more experience for garment fitters and/or devices that can improve the accuracy of the fitting process. While $78 \%$ of participants in this study reported that cost was not a barrier to replacing sleeves, for some, cost did limit the number of sleeves purchased each year. Participants with the financial resources and/or insurance coverage reported purchasing additional garments as a means to reduce burden (e.g., more garments to reduce the need for daily washing), improve comfort (e.g., different compression levels, sizes, or fabrics to accommodate fluctuations in lymphedema volume), and have more fashionable options (e.g., different colors to match outfits).

\section{Motivation}

The study findings suggest that efforts to support and enhance motivation to improve adherence to daytime compression is likely critical to long-term management of lymphedema. Although the vast majority of participants reported benefits from wearing the sleeve and negative consequences when they did not wear the sleeve, these incentives did not consistently influence adherence. Barriers such as discomfort, interference with function, and visibility of the garment were significant, and they were often reported as reasons for removal or non-wear of the sleeve. Besides efforts to address these barriers, healthcare provider approaches to increasing motivation could include developing wear-schedules and individual goals, planning strategies for times of non-wear, building in strategic "breaks", providing regular feedback on lymphedema status, and encouraging successful performance ${ }^{[27]}$.

Interventions to address psychosocial issues and negative emotions associated with daytime sleeve wear may include: (1) in person or virtual lymphedema support groups for sharing of issues and strategies; and (2) providing access to financial and psychosocial services ${ }^{[27]}$.

\section{Limitations}

There are a number of limitations to the research described in this paper. First, the study involved a convenience sample of women who previously took part in the LYNC trial. Thus, participants in this research may have been more motivated and better informed due to their prior involvement in a randomized controlled trial, and may not be a representative sample of women with BCRL. Second, lymphedema severity and perceived control were self-reported and based on participants' perception; thus, findings may not be an accurate representation of the status or control of their lymphedema. Third, the survey did not include questions on use of nighttime compression; however, in the focus group sessions, 20 of the 23 participants reported continued use of their night garment as a self-management 
strategy. Fourth, judgement when interpreting qualitative research is inevitably biased by the researchers' perspective. Although there may be different ways to interpret the data, we used a known framework and methodology to address this issue and to help us understand and better represent the findings. Fifth, very little research exists examining adherence to daytime compression, thus we were limited in our ability to make comparisons to findings of other studies.

In conclusion, the findings of this study suggest that, among past-participants of the LYNC trial, adherence to daytime compression sleeve use is less than optimal. While participants reported having good knowledge on the benefits of compression and the skills associated with use of a sleeve, numerous barriers to wear exist. The key themes related to barriers/non-use included discomfort, negative emotions, interference with function, social situations and visibility, and the use of alternative strategies. Further research is needed to explore the relative benefit of daytime compression and optimal wear times, as well as implementable strategies to improve adherence to daytime compression.

\section{DECLARATIONS}

\section{Authors' contributions}

Made substantial contributions to conception and design of the study and performed data analysis and interpretation: Al Onazi M, Dolgoy N, Parkinson J, McNeely ML

Performed data acquisition, as well as provided administrative, technical, and material support: Al Onazi M, Dolgoy N, Parkinson J, McNeely ML

\section{Availability of data and materials}

Data supporting the findings of the study will be deposited in a data repository at the University of Alberta.

\section{Financial support and sponsorship}

This work was supported by an Alberta Cancer Foundation Investigator Initiated Trials Grant and through funding from the Canadian Institutes of Health Research.

\section{Conflicts of interest}

All authors declared that there are no conflicts of interest.

\section{Ethical approval and consent to participate}

Ethical approval was obtained from the Health Research Ethics Board of Alberta: Cancer Committee for this additional follow-up component of the LYNC study. All participants provided amended informed consent.

\section{Consent for publication}

Not applicable.

\section{Copyright}

(c) The Author(s) 2020.

\section{REFERENCES}

1. DiSipio T, Rye S, Newman B, Hayes S. Incidence of unilateral arm lymphoedema after breast cancer: a systematic review and metaanalysis. Lancet Oncol 2013;14:500-15.

2. Hayes SC, Janda M, Cornish B, Battistutta D, Newman B. Lymphedema after breast cancer: incidence, risk factors, and effect on upper body function. J Clin Oncol 2008;26:3536-42.

3. McWayne J, Heiney SP. Psychologic and social sequelae of secondary lymphedema: a review. Cancer 2005;104:457-66.

4. Ostby PL, Armer JM. Complexities of adherence and post-cancer lymphedema management. J Pers Med 2015;5:370-88.

5. Paskett ED, Naughton MJ, McCoy TP, Case LD, Abbott JM. The epidemiology of arm and hand swelling in premenopausal breast cancer 
survivors. Cancer Epidemiol Biomarkers Prev 2007;16:775-82.

6. Moffatt C. International consensus: best practice for the management of lymphoedema. International Consensus London MEP Ltd 2006;3-52.

7. Chang CJ, Cormier JN. Lymphedema interventions: exercise, surgery, and compression devices. Semin Oncol Nurs 2013;29:28-40.

8. Armer JM, Hulett JM, Bernas M, Ostby P, Stewart BR, et al. Best practice guidelines in assessment, risk reduction, management, and surveillance for post-breast cancer lymphedema. Curr Breast Cancer Rep 2013;5:134-44.

9. Lasinski BB, McKillip Thrift K, Squire D, Austin MK, et al. A systematic review of the evidence for complete decongestive therapy in the treatment of lymphedema from 2004 to 2011. PM R 2012;4:580-601.

10. Ridner SH, Fu MR, Wanchai A, Stewart BR, Armer JM, et al. Self-management of lymphedema: a systematic review of the literature from 2004 to 2011. Nurs Res 2012;61:291-9.

11. McNeely ML, Campbell KL, Webster M, Kuusk U, Tracey K, et al. Efficacy of night-time compression for breast cancer related lymphedema (LYNC): protocol for a multi-centre, randomized controlled efficacy trial. BMC Cancer 2016;16:601.

12. Vignes S, Porcher R, Arrault M, Dupuy A. Factors influencing breast cancer-related lymphedema volume after intensive decongestive physiotherapy. Support Care Cancer 2011;19:935-40.

13. Cane J, O'Connor D, Michie S. Validation of the theoretical domains framework for use in behaviour change and implementation research. Implement Sci 2012;7:37.

14. Francis JJ, O’Connor D, Curran J. Theories of behaviour change synthesised into a set of theoretical groupings: introducing a thematic series on the theoretical domains framework. Implement Sci 2012;7:35.

15. Michie S, Johnston M, Francis J, Hardeman W, Eccles M. From theory to intervention: mapping theoretically derived behavioural determinants to behaviour change techniques. Applied Psychol 2008;57:660-80.

16. Michie S, van Stralen MM, West R. The behaviour change wheel: a new method for characterising and designing behaviour change interventions. Implement Sci 2011;6:42.

17. Atkins L, Francis J, Islam R, O’Connor D, Patey A, et al. A guide to using the Theoretical Domains Framework of behaviour change to investigate implementation problems. Implement Sci 2017;12:77.

18. Thorne S. Interpretive description: qualitative research for applied practice. New York: Routledge; 2016.

19. Thorne S, Kirkham SR, MacDonald-Emes J. Interpretive description: a noncategorical qualitative alternative for developing nursing knowledge. Res Nurs Health 1997;20:169-77.

20. Harris PA, Taylor R, Minor BL, Elliott V, Fernandez M, et al.; REDCap Consortium. The REDCap consortium: Building an international community of software platform partners. J Biomed Inform 2019;95:103208.

21. Harris PA, Taylor R, Thielke R, Payne J, Gonzalez N, et al. Research electronic data capture (REDCap)--a metadata-driven methodology and workflow process for providing translational research informatics support. J Biomed Inform 2009;42:377-81.

22. Braun V, Clarke V. Using thematic analysis in psychology. Qualitative Res Psychol 2006;3:77-101.

23. Moseley AL, Carati CJ, Piller NB. A systematic review of common conservative therapies for arm lymphoedema secondary to breast cancer treatment. Ann Oncol 2007;18:639-46.

24. Harris SR, Schmitz KH, Campbell KL, McNeely ML. Clinical practice guidelines for breast cancer rehabilitation: syntheses of guideline recommendations and qualitative appraisals. Cancer 2012;118:2312-24.

25. Brown JC, Cheville AL, Tchou JC, Harris SR, Schmitz KH. Prescription and adherence to lymphedema self-care modalities among women with breast cancer-related lymphedema. Support Care Cancer 2014;22:135-43.

26. Stuiver MM, Ten Tusscher MR, McNeely ML. Which are the best conservative interventions for lymphoedema after breast cancer surgery? BMJ 2017;357:j2330.

27. Fu MR, Ridner SH, Hu SH, Stewart BR, Cormier JN, et al. Psychosocial impact of lymphedema: a systematic review of literature from 2004 to 2011. Psychooncology 2013;22:1466-84. 